\title{
Effects of antenatal application of ambroxol and glucocorticoid on lung morphometry and signal transduction of bone morphogenetic protein in the fetal rat
}

\author{
XIAO-QING $\mathrm{CHEN}^{1}$, SHENG-HUA WU ${ }^{1}, \mathrm{XI}^{-R O N G ~ G U O}{ }^{2}$ and XIAO-YU ZHOU ${ }^{3}$ \\ ${ }^{1}$ Department of Pediatrics, The First Affiliated Hospital of Nanjing Medical University, Nanjing 210029; \\ ${ }^{2}$ Department of Pediatrics, Nanjing Maternal and Child Health Hospital of Nanjing Medical University, Nanjing 210004; \\ ${ }^{3}$ Department of the Neonate, Nanjing Children's Hospital of Nanjing Medical University, Nanjing 210008, P.R. China
}

Received January 2, 2012; Accepted April 10, 2012

DOI: $10.3892 / \mathrm{mmr} .2012 .888$

\begin{abstract}
Antenatal ambroxol, dexamethasone (Dex) and betamethasone (Beta) are used to prevent neonate respiratory distress syndrome. The present study aimed to investigate the role of ambroxol, Dex and Beta administered antenatally on lung morphogenesis and signal transduction of bone morphogenetic protein (BMP) in rat embryo. Fetal lungs treated with ambroxol, 1-day Beta, 3-day Dex and 3-day Beta were more mature compared to the controls as determined by light microscopy and transmission electron microscopy. Expression of BMP4 and bone morphogenetic protein receptor II (BMPR-II) mRNA was upregulated in the 1-dayBeta-, 3-day-Dex- and 3-day-Beta-treated animals. BMP4 and BMPR-II protein were significantly increased in the 1-dayBeta-, 3-day-Dex- and 3-day-Beta-treated animals. Ambroxol, Dex and Beta promoted the morphological development of rat fetal lung; Beta was more effective than Dex. A multi-dose of glucocorticoids exhited a more beneficial effect than a single dose. The effects of Beta and Dex may be mediated by regulation of BMP signal transduction in rat fetal lung.
\end{abstract}

Correspondence to: Dr Xiao-Yu Zhou, Department of the Neonate, Nanjing Children's Hospital of Nanjing Medical University, Nanjing 210008, P.R. China

E-mail: xyzhou161@163.com

Abbreviations: RDS, respiratory distress syndrome; Dex, dexamethasone; Beta, betamethasone; BMP4, bone morphogenetic protein 4; BMPR-II, bone morphogenetic protein receptor II; pSmad1, phospho-Smad1; 1d-Dex, 1-day-dexamethasone-treated; 1d-Beta, 1-day-betamethasone-treated; 3d-Dex, 3-day-dexamethasone-treated; 3d-Beta, 3-day-betamethasone-treated; GC, glucocorticoid; RT-PCR, reverse transcriptase polymerase chain reaction; GAPDH, glyceraldehye-3-phosphate dehydrogenase

Key words: ambroxol, glucocorticoid, lung, morphogenesis, bone morphogenetic protein

\section{Introduction}

Antenatal use of a glucocorticoid (GC) has proven beneficial in the prevention of neonatal respiratory distress syndrome (RDS) (1). Betamethasone (Beta) and dexamethasone (Dex) are routinely used to enhance fetal lung maturity. However, at present, no firm recommendations can be made regarding the choice of steroid (1). Ambroxol is a proven, reliable and effective expectorant. To date, there are no contraindications for the prenatal use of ambroxol (2). Ambroxol has been shown to significantly reduce RDS compared to placebo, without causing important adverse effects either on the mother or the baby. The potential harmful side effects of GCs have led to the testing of other drugs capable of accelerating fetal lung maturity, therefore ambroxol may be an alternative for the prevention of RDS (2-4).

It is now known that bone morphogenetic proteins (BMPs), which orchestrate extensive biological activity of multifunction, regulate the key steps of embryonic lung development (5-7). However, changes in the morphometric structure of the fetal rat lung following Dex, Beta and ambroxol antenatal administration, and whether GCs and ambroxol are involved in the BMP signal pathway remain unclear. Therefore, we investigated the ultramicrostructure of rat fetal lung and the molecular biological mechanism involving BMP signal transduction.

\section{Materials and methods}

Animals. All protocols were approved by the Animal Care and Use Committee of Nanjing Medical University. Eighteen Sprague-Dawley pregnant rats were randomly divided into 6 groups of 3 rats each. The rats in the control group, ambroxol group, 3-day-dexamethasone-treated (3d-Dex) group and 3-day-betamethasone-treated (3d-Beta) group received $4 \mathrm{ml}$ of saline or $100 \mathrm{mg} / \mathrm{kg} /$ day of ambroxol or $0.2 \mathrm{mg} / \mathrm{kg} / \mathrm{day}$ of Dex or $0.2 \mathrm{mg} / \mathrm{kg} / \mathrm{day}$ of betamethasone intraperitoneally injected on the 16th, 17th and 18th day of gestation, repectively. Rats in the 1-day-dexamethasone-treated (1d-Dex) group and the 1-daybetamethasone-treated (1d-Beta) group received either $0.2 \mathrm{mg} /$ $\mathrm{kg} / \mathrm{day}$ of Dex or Beta intraperitoneally on the 18th day of gestation, and both received $4 \mathrm{ml}$ of saline on the 16th and 17th day. 
Tissue collection and preparation. Cesarean sections were carried out on the 19th day of gestation. The right upper lobes of fetal lungs were obtained. Slices $(4 \mu \mathrm{m})$ were prepared for $\mathrm{H} \& \mathrm{E}$ staining for morphometric measurements and immunohistochemical analysis. Lung tissue was fixed in 5\% glutaraldehyde and preserved for transmission electron microscope observation. Several fetal lungs collected from the embryo were preserved at $-70^{\circ} \mathrm{C}$ for RT-PCR and western blotting.

Morphometric studies. The JD-801 color graphic pathological software (Jiangsu JEDA Science-Technology Development Co., Nanjing, China) was used to measure alveolar walls, respiratory thecal perimeter and alveolar equivalent diameter. Alveolar spherical surface area was calculated from a known alveolar equivalent circle radius, equal to $4 / 3 \pi r^{3}$. Lamellar bodies and ultrastructure were monitored under JEM-1010 transmission electron microscopy (JEM-1010; Jeol Electron Inc., Japan).

Transcriptase polymerase chain reaction ( $R T-P C R)$. RT-PCR was performed as previously described (8). In brief, after amplification, each product $(10 \mu \mathrm{l})$ was loaded on a $1.5 \%$ agarose gel for electrophoresis. Each experiment was performed in triplicate. The amplified products were quantified by UVP densitometric scanning (UVP Co., San Gabriel, CA, USA).

Immunohistochemistry. Immunohistochemistry was performed by a procedure described elsewhere (8). Tissue was incubated overnight with primary antibody in PBS at 1:250 dilution for goat anti-rabbit-BMP4 (Santa Cruz Biotechnology Inc., Santa Cruz, CA, USA) measurement, 1:500 dilution for mouse anti-goat BMPR-II (Becton, Dickinson and Company, NY, USA) measurement and 1:500 dilution for goat anti-rabbit phospho-Smad1 (pSmad1) measurement. The sections were incubated with 1:1,000 dilution of secondary antibody, i.e., sreptavidin-biotin complex (SABC; Wuhan Boster BIO-Engineering, Wuhan, China). After being washed, the sections were mounted with glycerol and digital images were acquired using microscopy (Olympus BH-3, Japan). Quantitative analyses were measured with JD-801 color graphic pathological software (Jiangsu JEDA ScienceTechnology Development Co.).

Western blotting. Western blots were carried out as previously described (8). Briefly, the proteins were extracted using Nuclear Cytosol Extraction kit and Membrane Cytosol Protein Extraction kit (KeyGEN Biotech, Nanjing, China). Protein from each sample $(80 \mu \mathrm{g})$ was separated by electrophoresis on an SDS-polyacrylamide gel and then transferred to a PVDF membrane. The membrane was incubated overnight at $4{ }^{\circ} \mathrm{C}$ with primary goat anti-rabbit antibody against BMP4 at 1:500 dilution, primary mouse anti-goat against BMPR-II at 1:500 dilution and rabbit anti-mouse against $\beta$-actin at 1:400 dilution (Santa Cruz Biotechnology Inc.). The membrane was then incubated at $37^{\circ} \mathrm{C}$ for $1 \mathrm{~h}$ with horseradish peroxidase-labeled rabbit anti-goat IgG antibody for BMP4 measurement, rabbit anti-mouse $\mathrm{IgG}$ antibody for BMPR-II measurement and anti-goat anti-rabbit IgG antibody for $\beta$-actin measurement at 1:1,000 final dilution. All the horseradish peroxidaselabeled antibodies were purchased from Beijing Biosynthesis Biotechnology Co. (Beijing, China). The target proteins were

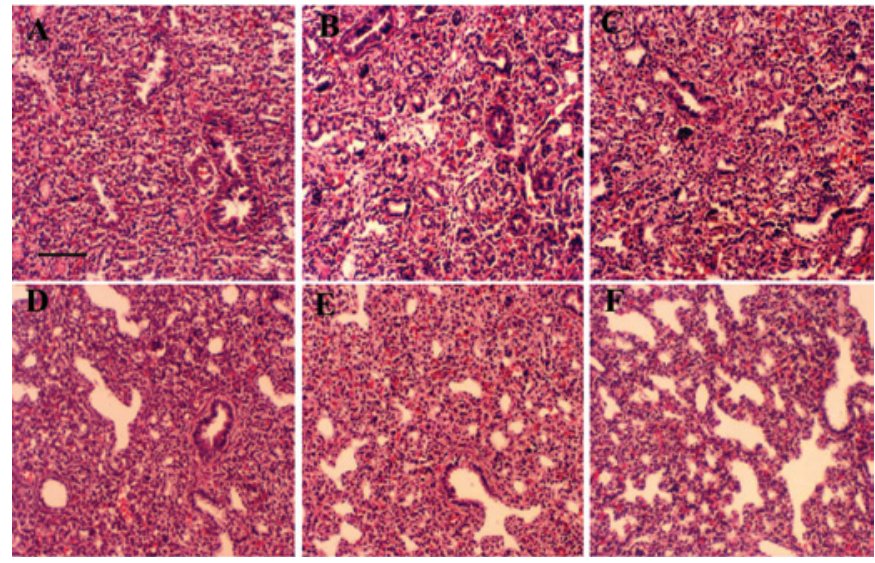

Figure 1. Light micrograph of H\&E-stained 5- $\mu \mathrm{m}$ sections of lung parenchyma (magnification, $\mathrm{x} 200$ ). More primitive alveoli were found in the (B) ambroxol group, (D) 1d-Beta group, (E) 3d-Dex group and (F) 3d-Beta group. (A) A higher volume of air space and lower interstitial tissue volume were noted in these groups as compared to the controls. Animals treated with (C) 1d-Dex and (A) saline had a lower volume of air space and thicker interstitial tissue. Scale bar, $50 \mu \mathrm{m}$.

visualized by X-ray autoradiograph and semiquantified by UVP-gel densitometry.

Statistical analyses. Quantitative data are expressed as the means \pm standard deviation (SD). Statistical comparisons were examined by ANOVA with Least Significant Difference (LSD) test. $\mathrm{P}<0.05$ denoted statistical significance.

\section{Results}

Lung morphometry. As shown in Figs. 1 and 2, more primitive alveoli were found in the fetal rats of the 1d-Beta, 3d-Beta, $3 \mathrm{~d}-$ Dex and ambroxl groups as compared to the controls. A higher volume of air space and lower interstitial tissue volume were noted in the above four groups as compared to these features in the controls. Animals of the 1d-Dex group showed a lower volume of air space and thicker interstitial tissue as compared to the controls.

As shown in Fig. 2, compared to the controls, alveolar walls in the fetal rats of the 3d-Beta, 3d-Dex, 1d-Beta and ambroxol groups were significantly thinner $(\mathrm{P}<0.05)$, while the respiratory thecal perimeter and alveolar space in the ambroxol, 1d-Beta, 3d-Beta and 3d-Dex groups were markedly larger $(\mathrm{P}<0.05)$. Larger respiratory thecal perimeter and alveolar space and thinner alveolar walls were found in the $3 \mathrm{~d}$-Beta group $(\mathrm{P}<0.01)$ as compared to the $3 \mathrm{~d}$-Dex group. Larger respiratory thecal perimeter and alveolar space and thinner alveolar walls were found in the $3 \mathrm{~d}$-Beta group $(\mathrm{P}<0.01)$ as compared to the 1d-Beta group. Larger respiratory thecal perimeter and alveolar space and thinner alveolar walls were found in the $3 \mathrm{~d}-$ Dex group $(\mathrm{P}<0.01)$ as compared to the $1 \mathrm{~d}-\mathrm{Dex}$ group, while no significant changes were found in the 1d-Dex group as compared to the controls ( $\mathrm{P}>0.05)$.

Ultrastructure of the pulmonary alveoli was studied using transmission electron microscopy. Lamellar bodies and cellular organs in the endochylema of type II alveolar cells, as indicated in Fig. 3, were more common in the 1d-Beta, 3d-Beta, 3d-Dex and ambroxol groups as compared to the 

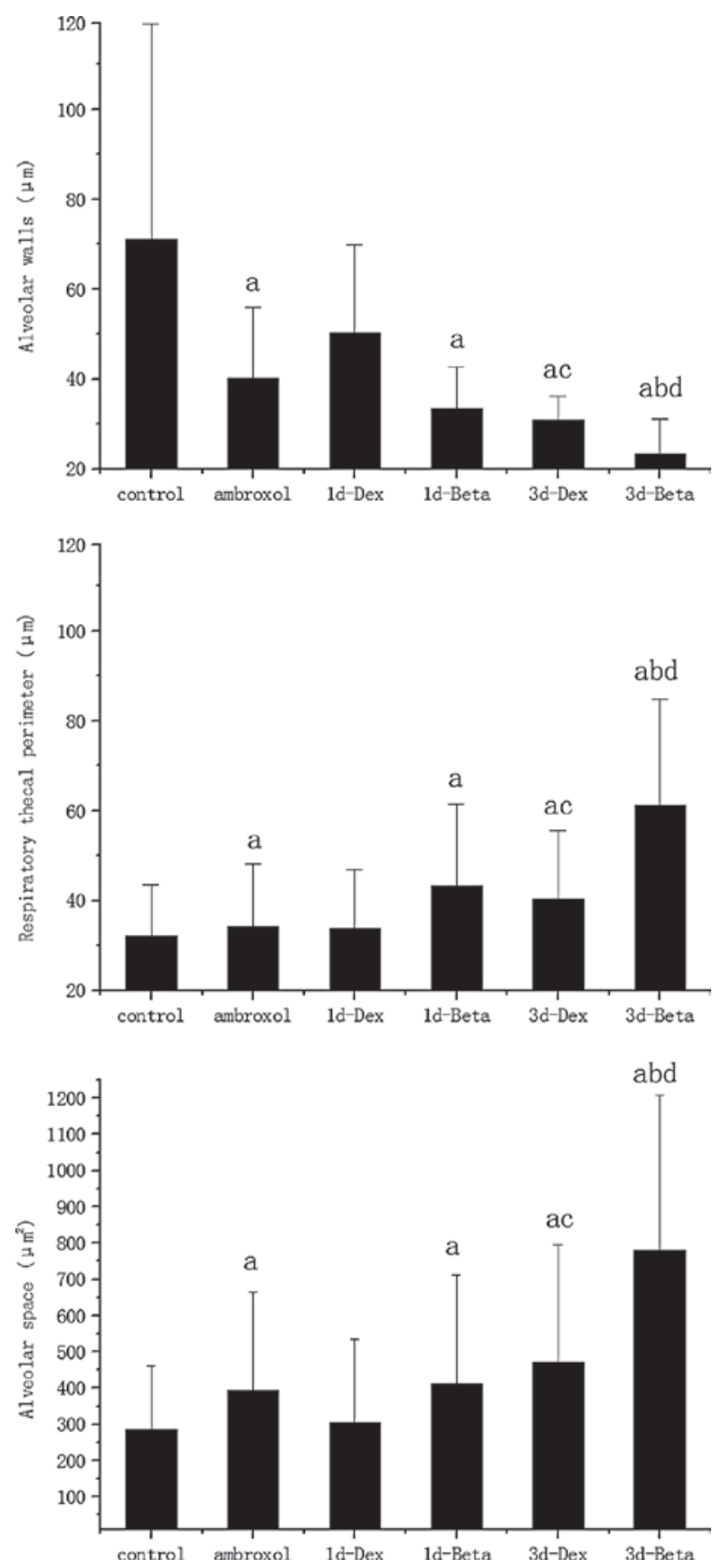

Figure 2. Comparisons of lung morphometry of fetal rats $(\bar{\chi} \pm s)$. Alveolar walls in fetal rats of the 3d-Beta, 3d-Dex, 1d-Beta and ambroxol groups were markedly thinner; respiratory thecal perimeter and alveolar space in the ambroxol, 1d-Beta, 3d-Beta and 3d-Dex groups were significantly larger than those of the controls $\left({ }^{a} \mathrm{P}<0.05\right)$. Larger respiratory thecal perimeter and alveolar space and thinner alveolar walls were found in the 3d-Beta group as compared to those of the $3 \mathrm{~d}$-Dex group $\left({ }^{\mathrm{b}} \mathrm{P}<0.01\right)$. Larger respiratory thecal perimeter and alveolar space and thinner alveolar walls were found in the $3 \mathrm{~d}$-Dex group ( $\mathrm{C}<0.01)$ as compared to those of the 1d-Dex group. Larger respiratory thecal perimeter and alveolar space and thinner alveolar walls were found in the 3d-Beta group as compared to those of the 1d-Beta group $\left({ }^{\mathrm{d}} \mathrm{P}<0.01\right)$, while no significant changes in alveolar wall thickness, respiratory thecal perimeter and alveolar space were found in the 1d-Dex group as compared to these values in the controls $(\mathrm{P}>0.05)$

controls. Significant changes were found in the fetal lung of the 3d-Beta and 3d-Dex groups. Empty bubble sample structure was existent in the 1d-Dex group and controls which had no lamellar body.

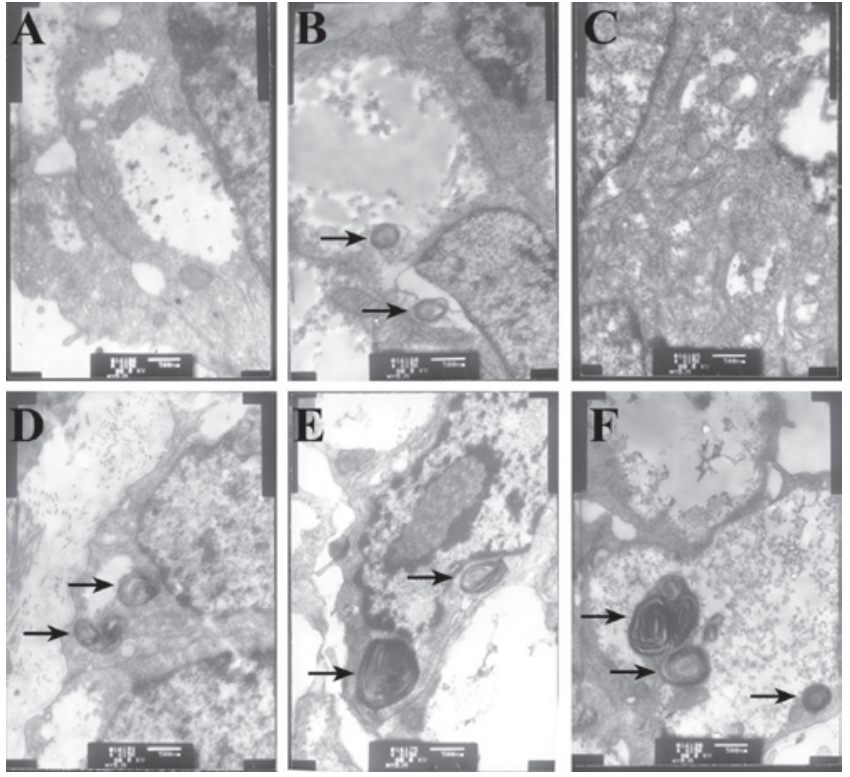

Figure 3. Electron microscopy images. Ultrastructure of the pulmonary alveolar lamellar bodies and cellular organs in the endochylema of type II alveolar cells were more common in the (B) ambroxol group, (D) 1d-Beta group, (E) 3d-Dex group and (F) 3d-Beta group, as compared to those of the controls (A). Significant changes were noted in the fetal lung of the $(F)$ 3d-Beta group and (E) 3d-Dex group. Lamellar bodies were not found in the (C) 1d-Dex group and (A) controls, while empty bubble sample structure was ubiquitous. Arrows indicate lamellar bodies. Scale bars, $500 \mathrm{~nm}$ for all images. Magnification, x15,000.

$R T$-PCR. As shown in Fig. 4, BMP4 mRNA expression was higher in the $3 \mathrm{~d}$-Beta $(\mathrm{P}<0.01), 3 \mathrm{~d}$-Dex and $1 \mathrm{~d}-$ Beta groups $(\mathrm{P}<0.05)$, as compared to the controls. No significant changes were observed between the ambroxol and 1d-Dex groups $(\mathrm{P}>0.05)$. BMPR-II expression was upregulated in the 1d-Dex, 1d-Beta, 3d-Dex $(\mathrm{P}<0.05)$ and 3d-Beta groups $(\mathrm{P}<0.01)$, as compared to the controls. However, no changes were found in the ambroxol group in contrast to those of controls $(\mathrm{P}>0.05)$.

\section{Immunohistochemistry}

BMP4 expression. As presented in Fig. 5, BMP4 was localized mainly on the endochylema of the respiratory epithelium of small and medium bronchi. Expression of BMP4 in the 1d-Beta, 3d-Dex and 3d-Beta groups was higher than that of the controls $(\mathrm{P}<0.05)$. However, no obvious change was detected in the 1d-Dex and ambroxol groups as compared to the controls $(\mathrm{P}>0.05)$.

BMPR-II expression. As shown in Fig. 6, BMPR-II protein was mostly expressed in the membrane of the epithelial and bronchial cells, and was markedly higher in the 1d-Beta, 1d-Dex, $3 d-D e x$ and $3 d-B e t a$ groups as compared to the controls $(\mathrm{P}<0.01)$. However, there was no difference between the ambroxol group and controls.

PSmadl expression. As shown in Fig. 7, pSmad1 protein existed primarily in the cytoplasm of bronchial and aleolar epithelial and mesenchyma cells of embryo rat lung. pSmad1 was upregulated in the 1d-Beta, 3d-Dex and 3d-Beta groups $(\mathrm{P}<0.01)$ as compared to the controls. No significant changes in pSmad1 expression were found among the 1d-Dex, ambroxol and control groups $(\mathrm{P}>0.05)$. 

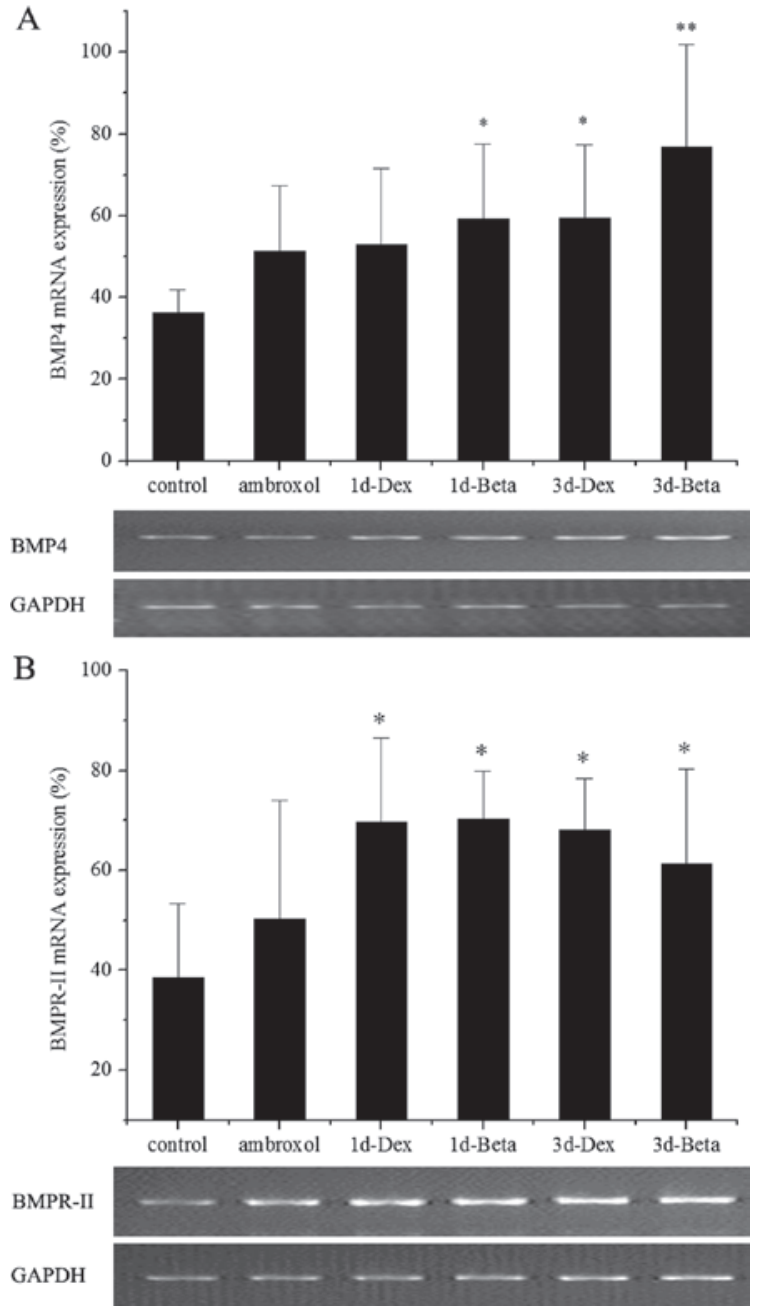

Figure 4. Expression of BMP4 and BMPR-II mRNA. (A) Expression of BMP4 mRNA was upregulated in the 1d-Beta, 3d-Dex and 3d-Beta groups as compared to the controls. No significant changes were found in the ambroxol and 1d-Dex groups compared to the controls. (B) Expression of BMPR-II mRNA was upregulated in the 1d-Dex, 1d-Beta, 3d-Dex and 3d-Beta groups as compared to the controls. Changes in BMP4 and BMPR-II mRNA expression were not obvious in the ambroxol group in contrast to those of the controls. $\mathrm{P}<0.01$ and ${ }^{* *} \mathrm{P}<0.05$.

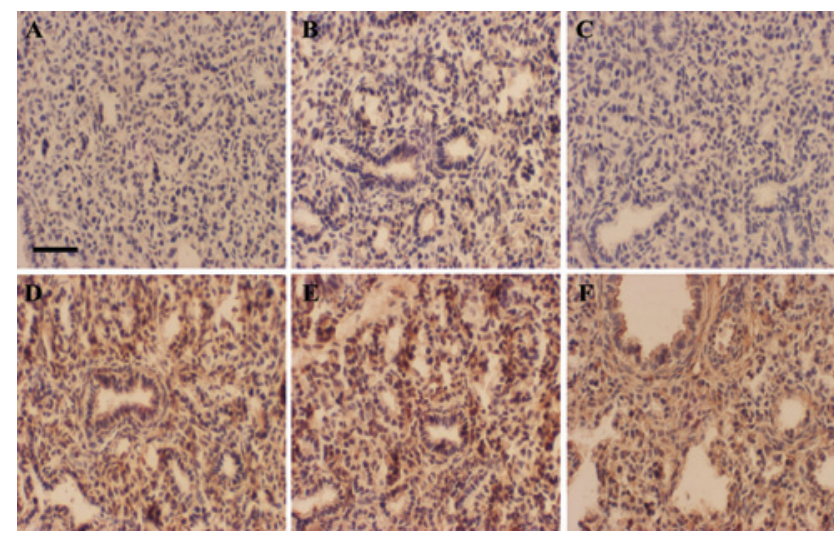

Figure 5. Expression of BMP4 protein in rat fetal lung as detected with immunohistochemical staining. (A-F) Expression in controls, the ambroxol, 1d-Dex, 1d-Beta, 3d-Dex and 3d-Beta groups, respectively. BMP4 protein was mostly expressed in the membrane of the epithelial and bronchial cells as shown under microscopy. Compared to the controls (A), BMP4 expression was upregulated in the (C) 1d-Dex group, (D) 1d-Beta group, (E) 3d-Dex group and (F) 3d-Beta group, while no difference in expression of BMP4 was noted in the ambroxol group. Scale bar, $25 \mu \mathrm{m}$; magnification, $\mathrm{x} 400$.
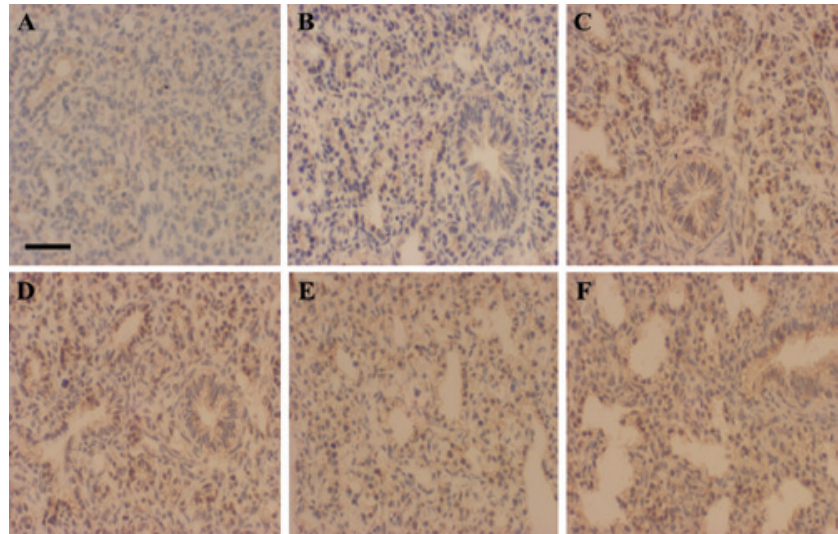

Figure 6. Expression of BMPR-II protein in rat fetal lung as detected with immunohistochemical staining. (A-F) Expression in controls, the ambroxol, 1d-Dex, 1d-Beta, 3d-Dex and 3d-Beta groups, respectively. BMPR-II protein was mostly expressed in the membrane of the epithelial and bronchial cells as shown under microscopy. Compared to the controls (A), BMPR-II expression was upregulated in the (C) 1d-Dex group, (D) 1d-Beta group, (E) 3d-Dex group and (F) 3d-Beta group, while no difference in expression of BMPR-II was noted in the ambroxol group. Scale bar, $25 \mu \mathrm{m}$; magnification, $\mathrm{x} 400$.
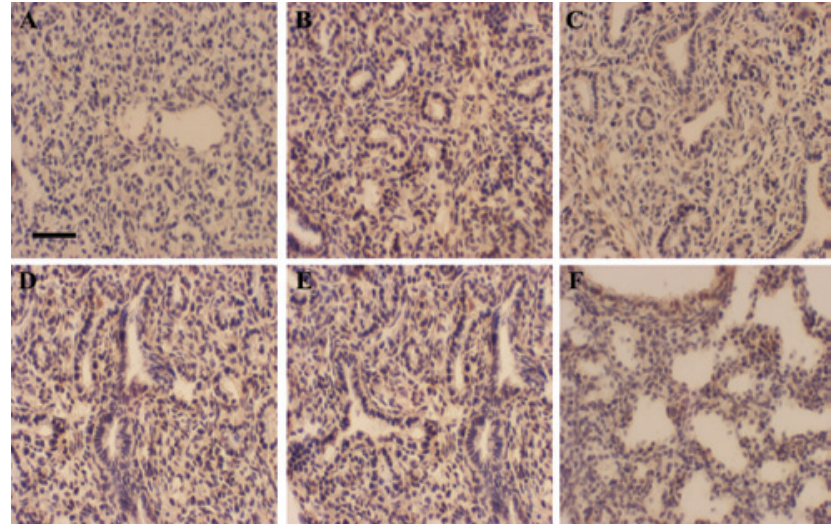

Figure 7. Expression of pSmad1 protein in rat fetal lung as detected with immunohistochemical staining. (A-F) Expression in controls, the ambroxol, 1d-Dex, 1d-Beta, 3d-Dex and 3d-Beta groups, respectively. pSmad1 protein was mostly expressed in the membrane of the epithelial and bronchial cells as shown under microscopy. Compared to the controls (A), pSmad1 expression was upregulated in the (C) 1d-Dex group, (D) 1d-Beta group, (E) 3d-Dex group and (F) 3d-Beta group, while no difference in expression of pSmad1 was noted in the ambroxol group. Scale bar, $25 \mu \mathrm{m}$; magnification, $\mathrm{x} 400$.

Western blot analysis. As shown in Fig. 8, expression of BMP4 was higher in the 1d-Beta, 3d-Dex and 3d-Beta groups as compared to that of the controls $(\mathrm{P}<0.01)$. However, our present study showed no significant difference among the ambroxol, the 1d-Dex group and controls ( $\mathrm{P}>0.05)$. Compared to that of controls, the expression of BMPR-II in the 1d-Dex, 1d-Beta, $3 \mathrm{~d}-$ Dex and $3 \mathrm{~d}-\mathrm{Beta}$ groups was markedly higher $(\mathrm{P}<0.01)$, while no difference was noted in the ambroxol group.

\section{Discussion}

Neonatal RDS is a serious complication of premature infants and is the main cause of neonatal mortality and disability. It is crucial for pregnant women with high-risk pregnancies to receive GC, including Dex and Beta, to promote infant pre-natal lung development and reduce perinatal mortality. 


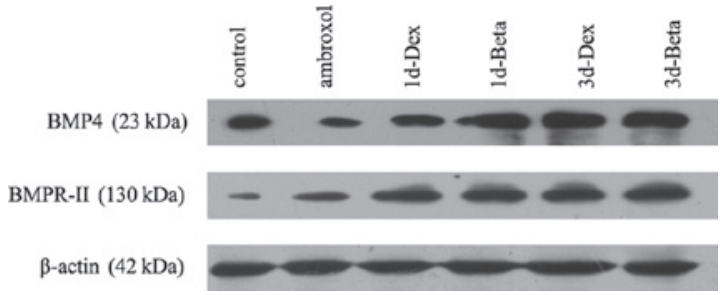

Figure 8. Expression of BMP4 and BMPR-II in the rat fetal lung as detected by western blotting. Significantly higher expression of BMP4 was found in the 1d-Beta, 3d-Dex and 3d-Beta groups as compared to that of the controls. In addition, the expression of BMPR-II in the 1d-Dex, 3d-Dex, 1d-Beta and $3 \mathrm{~d}$-Beta groups was markedly higher $(\mathrm{P}<0.01)$, but not in the ambroxol group, compared to the controls.

Beta preventive therapy is associated with a reduced risk for neonatal death, showing trends of significant decreased risk for intraventricular hemorrhage and severe retinopathy of prematurity and a significantly lower rate of pulmonary complications compared to Dex therapy $(9,10)$. It may be in the best interest of neonates to receive treatment with Beta rather than Dex when available $(9,11,12)$. Multiple antenatal courses of Dex have been associated with an increased risk of leukomalacia and 2-year infant neurodevelopmental abnormalities in contrast to Beta (13). Antenatal Beta is the best choice for treatment of women at risk of preterm delivery (11). A system review suggests that Dex may have some benefits compared to Beta, such as less intraventricular hemorrhage, but few additional conclusions about optimal antenatal corticosteroid regimens were able to be made (14). Nevetheless, at present, no firm recommendations can be made regarding choice of steroid (15).

Recently, the potential danger of large doses of maternal hormone therapy caused extensive attention. The pharmacology of corticosteroids suggests that a lower total GC dose may be as effective as the current treatment recommendations $(11,16)$. Therefore, to compare the diversity of morphological structures of fetal lung following the therapy of antenatal use of Dex and Beta and to reduce GC administration side effects as much as possible, we used a low dose $(0.2 \mathrm{mg} / \mathrm{kg})$ of medication as compared to previous studies (17-19). Two drugs were used at the same doses and in the same method of treatment. In the present study, light microscopy, electron microscopy and image analysis results indicated that small doses of GC effectively promoted fetal lung maturity. Our study confirmed that the effect of Beta was more efficient than that of Dex in fetal lung development (Figs. 1-3). The most striking changes following GC treatment were found in the 3d-Dex and 3d-Beta groups compared to those of the 1d-Dex and 1d-Beta groups. Therefore, we hypothesized that improved respiratory function after antenatal GC administration was due to structural maturity within the rat fetal lung.

Ambroxol is a relatively new promoter of fetal lung maturation acting on alveolar cells type II, by promoting indirect synthesis and release of surfactant; however, the specific mechanism is not known (20). Application of ambroxol may be a safe and effective option for pregnant women with a fetus at risk of infant RDS (21). In addition, the incidences of bronchopulmonary dysplasia, intraventricular hemorrhage and post-natally acquired pneumonia were significantly reduced in the ambroxol group compared to the control. There were no side effects attributable to the drug (22). However, another study did not find evidence for the efficacy of antenatal ambroxol treatment for the prevention of neonatal respiratory distress syndrome (23).

To date, it is not known whether or not ambroxol antenatal application to pregnant rats affects fetal lung development. In the present study, we also compared ambroxol administration to GCs and controls. Ambroxol adequately promoted the development of the fetal lung as GC, since larger respiratory thecal perimeter, alveolar space and thinner alveolar walls were found in the ambroxol group (Fig. 1). In addition, electron microscopy indicated that the pulmonary alveolar lamellar bodies and cellular organs in endochylema of type II alveolar cells were more common in the ambroxol group (Fig. 3). Even though light microscopy, electron microscopy and image analysis results indicated that the effect of GC was more beneficial than that of ambroxol in morphometry, ambroxol may be a suitable choice of antenatal preventive administration to pregnant women with contraindications who were administered GC over 1 week without delivery or complicated by diabetes, high blood pressure and infection.

BMPs play multiple roles in lung development. However, the precise roles of these molecules and how they interact in the developing lung are subject to debate $(5,6)$. BMPs are multi-functional growth factors that belong to the TGF- $\beta$ superfamily. BMP-activated Smads are the canonical intracellular signaling proteins that transduce the BMP signal from the cell surface to nuclei, wherein gene expression is regulated (24). The BMP type II receptor binds several BMPs and mediates BMP signaling. Signal transduction studies have revealed that Smad1, 5 and 8 are the immediate downstream molecules of BMP receptors and play a central role in BMP signal transduction $(25,26)$.

Despite of the widely acknowledged significance of BMP signaling in embryonic lung development, changes in the BMP pathways in fetal lung has not been demonstrated. The present study, therefore, identified whether antenatal administration of ambroxol and GC altered the expression of members of BMPs. RT-PCR and immunohistochemistry showed that expression levels of BMP4 mRNA and protein were upregulated in the 1d-Beta, 3d-Dex and 3d-Beta groups (Figs. 4 and 5). Western blot analysis confirmed the expression of the active form of mature BMP4 protein (Fig. 8). The results of the detection of BMP4 protein expression in each group were in agreement with the immunohistochemical findings. We speculate that prenatal application of Beta and Dex enhanced BMP4 expression in fetal lung. However, there was no change in BMP4 expression between the 1d-Dex group and controls, which may be attributed to the low dosage and the short regimen of Dex. Similar treatment results were found in the expression of BMPR-II, as shown in Figs. 4, 6 and 8. It has been suggested that GCs may activate the BMP signaling pathway, promoting ligands and its combination with the receptor and intracellular signal transmission.

As indicated in Fig. 7, expression of pSmad1, the critical effector that controls transcriptional responses of BMP signaling, was found upregulated in the GC groups, particularly in the 3d-Beta group. Consequently, it is reasonable to speculate that GC may activate Smad1 by upregulation of 
BMP and combination of BMP with BMPR-II, thus affecting endonuclear target gene transcription and protein expression, which is involved in the regulation of embryonic lung development.

Our experiment also showed that expression levels of BMP4, BMPR-II and Smad1, members of the BMP signaling pathways, were not altered after antenatal ambroxol administration neither at the gene nor protein level (Figs. 4-8). It has been suggested that antenatal ambroxol treatment does not alter BMP signal transduction pathways of molecular expression. The mechanism of ambroxol treatment in lung development may involve other signaling pathways and require further exploration.

In conclusion, prenatal administration of ambroxol and low doses of Dex and Beta promote morphological structure maturity in the rat fetal lung. This study demonstrated that Beta and Dex intervention significantly activated expression of BMP signaling pathway members BMP4, BMPR-II and Smad1 in lung tissue at both the gene transcription and protein level, which may be an importment mechanism of lung maturation. Therefore, our study elucidates the mechanism by which antenatal use of GC and ambroxol promotes lung development and provides a novel antenatal preventative treatment for neonatal RDS.

\section{Acknowledgements}

The authors are grateful to Professor Xi-Rong Guo for the critical advice on the design of the study. They also thank Mei Guo for the assistance in the graphical analysis.

\section{References}

1. Roberts D and Dalziel S: Antenatal corticosteroids for accelerating fetal lung maturation for women at risk of preterm birth. Cochrane Database Syst Rev 3: CD004454, 2006.

2. Vytiska-Binstorfer E, Salzer H and Simbruner G: Ambroxol in comparison with betamethasone for the stimulation of antepartal lung maturity. A clinical double-blind study. Zentralbl Gynakol 108: 220-229, 1986 (In German).

3. Cosmi EV and Di Renzo GC: Prevention and treatment of fetal lung immaturity. Fetal Ther 4 (Suppl 1): 52-62, 1989.

4. Kimya Y, Küçükkömürcü S, Ozan H and Uncu G: Antenatal ambroxol usage in the prevention of infant respiratory distress syndrome. Beneficial and adverse effects. Clin Exp Obstet Gynecol 22: 204-211, 1995.

5. Pongracz JE and Stockley RA: Wnt signalling in lung development and diseases. Respir Res 7: 15-25, 2006.

6. Warburton D, Bellusci S, De Langhe S, et al: Molecular mechanisms of early lung specification and branching morphogenesis. Pediatr Res 57: 26-37, 2005.

7. Cardoso WV and Lü Jining: Regulation of early lung morphogenesis: questions, facts and controversies. Development 133: 1611-1624, 2006.

8. Chen XQ, Wu SH and Zhou XY: Effects of antenatal administration of dexamethasone and betamethasone on signal transduction of bone morphogenetic protein in the fetal lungs of rats. Zhongguo Dang Dai Er Ke Za Zhi 12: 891-896, 2010 (In Chinese)
9. Feldman DM, Carbone J, Belden L, Borgida AF and Herson V: Betamethasone vs dexamethasone for the prevention of morbidity in very low birthweight neonates. Am J Obstet Gynecol 197: 284. e1-4, 2007.

10. Lee BH, Stoll BJ, McDonald SA and Higgins RD: National Institute of Child Health and Human Development Neonatal Research Network. Adverse neonatal outcomes associated with antenatal dexamethasone versus antenatal betamethasone. Pediatrics 117: 1503-1510, 2006.

11. Jobe AH and Soll RF: Choice and dose of corticosteroid for antenatal treatments. Am J Obstet Gynecol 190: 878-881, 2004.

12. Ozdemir H, Guvenal T, Cetin M, Kaya T and Cetin A: A placebo-controlled comparison of effects of repetitive doses of betamethasone and dexamethasone on lung maturation and lung, liver, and body weights of mouse pups. Pediatr Res 53: 98-103, 2003.

13. Spinillo A, Viazzo F, Colleoni R, Chiara A, Maria Cerbo R and Fazzi E: Two-year infant neurodevelopmental outcome after single or multiple antenatal courses of corticosteroids to prevent complications of prematurity. Am J Obstet Gynecol 191: 217-224, 2004.

14. Brownfoot FC, Crowther CA and Middleton P: Different corticosteroids and regimens for accelerating fetal lung maturation for women at risk of preterm birth. Cochrane Database Syst Rev 8: $1-58,2008$.

15. Sweet DG, Carnielli V, Greisen G, et al: European Association of Perinatal Medicine. European consensus guidelines on the management of neonatal respiratory distress syndrome in preterm infants - 2010 update. Neonatology 97: 402-417, 2010.

16. Coe CL and Lubach GR: Developmental consequences of antenatal dexamethasone treatment in nonhuman primates Neurosci Biobehav Rev 29: 227-235, 2005.

17. Bortolin L, Boer M, Christiansen S, García Rivello H, Arbat J and Fustiñana C: Effects of prenatal steroids in the development of necrotizing enterocolitis in Wistar rat neonates. Arch Argent Pediatr 109: 24-29, 2011.

18. Arima M, Kumai T, Asoh K, et al: Effects of antenatal dexamethasone on antioxidant enzymes and nitric oxide synthase in the rat lung. J Pharmacol Sci 106: 242-248, 2008.

19. Remesal A, San Feliciano L, Isidoro-García M and Ludeña D: Effects of antenatal betamethasone and dexamethasone on the lung expression of vascular endothelial growth factor and alveolarization in newborn rats exposed to acute hypoxia and recovered in normoxia or hyperoxia. Neonatol 98: 313-320, 2010.

20. Carrera G, Liberatore A, Riboni G and Clementi M: Surfactant and ambroxol in the therapy of idiopathic respiratory syndrome in newborn infants. Minerva Pediar 43: 723-730, 1991.

21. Wauer RR, Schmalisch G, Bohme B, Arand J and Lehmann D: Randomized double blind trial of ambroxol for the treatment of respiratory distress syndrome. Eur J Pediatr 151: 357-363, 1992.

22. Laoag-Fernandez JB, Fernandez AM and Maruo T: Antenatal use of ambroxol for the prevention of infant respiratory distress syndrome. Obstet Gynaecol Res 26: 307-312, 2000.

23. Dani C, Grella PV, Lazzarin L and Rubaltelli FF: Antenatal ambroxol treatment does not prevent the respiratory distress syndrome in premature infants. Eur J Pediatr 156: 392-393, 1997.

24. Derynck R and Zhang YE: Smad-dependent and Smadindependent pathways in TGF- $\beta$ family signaling. Nature 425 : 577-584, 2003

25. Chen D, Zhao M and Mundy GR: Bone morphogenetic proteins. Growth Factors 22: 233-241, 2004.

26. Morrell NW: Pulmonary hypertension due to BMPR2 mutation: a new paradigm for tissue remodeling? Proc Am Thorac Soc 3: 680-686, 2006. 